Article

\title{
Polysialic Acid Modified Liposomes for Improving Pharmacokinetics and Overcoming Accelerated Blood Clearance Phenomenon
}

\author{
Xi Han ${ }^{1,2}$, Ting Zhang ${ }^{1,2}$, Mengyang Liu ${ }^{1}$, Yanzhi Song ${ }^{1}$, Xinrong Liu ${ }^{1}$ and Yihui Deng ${ }^{1, *}$ \\ 1 College of Pharmacy, Shenyang Pharmaceutical University, Shenyang 110016, China; \\ m13588897912@163.com (X.H.); zhangting55@163.com (T.Z.); liumengyang@syphu.edu.cn (M.L.); \\ songyanzhi@syphu.edu.cn (Y.S.); 13898891331@163.com (X.L.) \\ 2 College of Pharmaceutical Science, Zhejiang Chinese Medical University, Hangzhou 310053, China \\ * Correspondence: dengyihui@syphu.edu.cn; Tel.: +86-024-43520553
}

Received: 4 August 2020; Accepted: 26 August 2020; Published: 28 August 2020

\begin{abstract}
Poly (ethylene glycol) (PEG) modified nanocarriers are being used widely in the drug delivery system (DDS). However, the "accelerated blood clearance (ABC) phenomenon" was induced upon repeated administration of PEG-modified liposomes, resulting in reduced blood circulation time, and increased accumulation in liver and spleen. To avoid the unexpected phenomenon, polysialic acid (PSA) was selected to modify liposomes. PSA is a natural, highly hydrophilic polysaccharide polymer for which no receptors exists in the body. It is non-immunogenic, biodegradable and endows the conjugated bioactive macromolecule and drugs with increased circulation time in vivo. In the present study, the in vivo evaluation showed that PSA modified liposomes (PSA-Lip) afford extended blood circulation in wistar rats and beagle dogs. Moreover, the ABC phenomenon did not occur and the IgM antibody was not induced after repeated injections of PSA-Lip. These results strongly suggest that PSA modification represents a promising strategy to afford good stealth of the liposomes without evoking the $\mathrm{ABC}$ phenomenon.
\end{abstract}

Keywords: polysialic acid; poly (ethylene glycol); extended blood circulation; accelerated blood clearance phenomenon

\section{Introduction}

It is well known that common nanocarriers are easily recognized by the mononuclear phagocyte system (MPS), and are therefore rapidly cleared from the blood circulation, distributed in the liver and spleen, which affect the effectiveness. In the development history of the drug delivery system (DDS), the PEGylation technology of drug molecules and carriers has a landmark significance [1,2]. PEGylation technology can reduce the recognition of drugs/carriers by MPS and prolong blood circulation time, thereby using the enhanced permeability and retention (EPR) effect to achieve disease sites targeting [3,4]. PEGylation technology has many advantages such as prolonging circulation time, improving water solubility and increasing stability. The application of PEGylation technology in many aspects has been approved by the FDA for clinical use [5].

Liposomes are phospholipid bilayer vesicles with an aqueous phase inside. Because of this special constitution, liposomes could encapsulate both hydrophilic and hydrophobic drugs. Liposomes are widely used in medicine and other fields, owing to their unique characteristic, such as biodegradability and biocompatibility, improving the therapeutic index and reducing systemic toxicity [6,7]. However, conventional liposomes have the problems of poor colloidal stability, and rapid clearance from the blood by MPS [8,9]. Polyethylene glycol (PEG), the hydrophilic, neutral polymers, could increase the hydrophilicity and provide a hydration layer on the liposome surface, which help the modified 
liposomes decrease recognition by the MPS, and prolong circulation time in vivo after intravenous injection [10]. It has been thought that PEG could serve as the gold standard material in the drug carrier construction [11,12]. Nevertheless, it has been pointed out that the PEGylated liposomes would be cleared from blood quickly and gathered in the liver and spleen after an interval of several days when the second dose was administered intravenously. This is called "accelerated blood clearance (ABC) phenomenon" [13,14].

It was reported that $\mathrm{ABC}$ phenomenon contained sequential events, the first injected dose of PEGylated liposomes induced anti-PEG IgM antibody production in the spleen; the second dose of PEGylated liposomes were quickly recognized and bound by the IgM antibody, and the complement was activated by C3 fragments opsonization and enhanced uptake by MPS [15]. The long circulation advantages of PEGylated liposomes would be greatly decreased by the $\mathrm{ABC}$ phenomenon. If the PEGylated liposomes encapsulated cytotoxicity drug, the ABC phenomenon would causes serious toxic effects due to the accumulation in MPS in liver and spleen [16]. In addition, it has been reported that acute hypersensitivity reactions was caused by PEGylated liposomes after injections in some individuals. In the hypersensitive individuals the plasma SC5b-9 was significantly increased after injections of PEG modified liposomes [17]. Therefore, the hypersensitivity reactions was corrected with complement activation. In order to solve the ABC phenomenon of PEGylation technology, researchers have changed the dosing interval, the modification density of PEG derivatives, and the physicochemical properties of PEG preparations $[13,18,19]$. However, most of these approaches did not eliminate the ABC phenomenon completely and therapeutic efficacy of PEGylated liposomes was weakened. Taken together, as long as PEG exists in the drug carrier construction, the ABC phenomenon is inevitable. Therefore, seeking an alternative approach remains extremely urgent by extending the circularly stability of modified liposomes and avoiding the $\mathrm{ABC}$ phenomenon.

Polysialic acid (PSA), composed of a-2,8-linked 5-N-acetylneuraminic acid (Neu5Ac), is a highly hydrophilic polysaccharide in mammalian tissue. PSA is an endogenous substance, which is non-toxic, non-immunogenic and bio-degradable [20,21]. PSA is a post-translational modification of neural cell adhesion molecule (NCAM) [22]. Studies have shown that [23] PSA would increase the hydrodynamic radius of modified NCAM, improve the repulsive ability between NCAM molecules, and PSA also weakened the adhesion force between modified NCAM and other molecules. In addition, PSA in microorganisms is consistent with the structure and immunogenicity of PSA in mammals [24]. Neisseria will use PSA to wrap themselves and recruit $\mathrm{H}$ factor to prevent complement $\mathrm{C}$ fragments from depositing on cell surfaces, inhibit the formation of attack complexes, mask potential epitopes and reduces recognition by the immune system $[25,26]$. Therefore, PSA can escape immune system surveillance through immune camouflage. Meanwhile, the repellent layer of PSA will weaken the recognition of opsonins and reduce MPS uptake. Based on the above properties, PSA can be suitably applied to long-circulation drug delivery system $[27,28]$. Some studies on PSA modified liposome-based drug delivery system have been reported by our group. In our previous work [29], PSA-polyethylene glycol (PSA-PEG) conjugate was synthesized for the decoration of liposomal epirubicin. The liposomal formulations had good storage and dilution stability. In vivo antitumor studies revealed that PSA-PEG modified liposomal epirubicin, serving as a binding molecule to the tumor site, exhibited increased accumulation in tumor area, enhanced the antitumor activity with reduced systemic toxicity. Luo et al. [30] synthesized a PSA-p-octadecylamine conjugate (PSA- $p$-ODA) and decorate it on the surface of liposomal pixantrone, Experimental results showed that the modification of PSA- $p$-ODA could prolong the circulation time of the liposomal pixantrone, and targeted to peripheral blood neutrophils, which provide a neutrophil-mediated drug delivery system for the eradication of tumors.

In previous study, the PSA derivative was synthesized with octadeyl dimethyl betaine (BS18) and the synthetic product PSA-BS18 was modified on the liposomes (PSA-Lip) [31]. The PSA-Lip presented extended residence in the blood circulation. In the present study, the systemic study was conducted to 
investigate whether PSA-Lip would overcome the ABC phenomenon and complement activation after intravenous administrations.

\section{Materials and Methods}

\subsection{Materials}

Hydrogenated soy phosphatidylcholine (HSPC) and 1,2-dioctadecanoyl-sn-glycero-3-phospho(1'-rac-glycerol) (sodium salt) (DSPG) was got from Lucas Meyer (Düsseldorf, Germany). 1,2-Distearoylsn-glycero-3-phosphoethanolamine- $N$-[methoxy (polyethylene glycol)-2000] (mPEG2000-DSPE) and cholesterol $(\mathrm{CH})$ were obtained from Shanghai Advanced Vehicle Technology Pharmaceutical L.T.D. (Shanghai, China). 1,1'-dioctadecyl-3,3,3',3'-tetramethyl indotricarbocyanine Iodide (DiR) was purchased from American ATT Bioquest, Inc (Sunnyvale, CA, USA). Sephadex ${ }^{\circledR}$ G-50 was purchased from Pharmacia Biotech Inc. (Piscataway, NJ, USA). Polysialic acid-octadecyl dimethyl betaine (PSA-BS18) was synthesized in our laboratory [31].

\subsection{Animals}

Male wistar rats, weighting 180-220 g and male beagle dogs, weighting 10-12 kg were provided by the Laboratory Animal Center of Shenyang Pharmaceutical University (Shenyang, China). All animal experiments are in compliance with animal ethics committee standards [32]. Laboratory Animal Center of Shenyang Pharmaceutical University, the approval number is: IACUC-C2018-6-11-202.

\subsection{Synthesis of PSA-BS18}

PSA-BS18 was synthesized by combining the hydroxyl groups of PSA with the carboxyl group of BS18. EDC/NHS was used as a catalyst; the synthetic study was reported previously [31].

\subsection{Preparation of Liposomes}

Liposomes were prepared by the lyophilized hydration method [33]. For the common liposomes (CLip), HSPC, DSPG, CH were dissolved in the organic solvent tert-butanol, 3:1:2 (mol/mol), and PEGylated liposomes (PEG-Lip), HSPC, DSPG, CH and mPEG2000-DSPE were dissolved in the organic solvent tert-butanol, 3:1:2:0.32 (mol/mol). For PSA modified liposomes (PSA-Lip), HSPC, DSPG and CH were dissolved in the organic solvent tert-butanol, 3:1:2 (mol/mol). PSA-BS18 was added in water phase. The water phase was then injected into the lipid solution phase at $60{ }^{\circ} \mathrm{C}$. The obtained lipid solution was freeze-dried. Then $5 \%$ glucose was added to the lyophilized cake for hydration under continuous agitation at $60^{\circ} \mathrm{C}$ for $20 \mathrm{~min}$. The hydration solution was sonicated by an ultrasonic cell pulverizer for $200 \mathrm{~W}, 2 \mathrm{~min}$ and $400 \mathrm{~W}, 6 \mathrm{~min}$. At last, the liposome suspension passed through $0.45-$, and $0.22-\mu \mathrm{m}$ microporous filters in sequence.

In the preparation of DiR labeled CLip, PSA-Lip and PEG-Lip, DiR was added in the mixture solution before the lyophilization, and the other process was as same as mentioned above.

\subsection{Characterization of Liposomes}

\subsubsection{Particle Size, PDI and Zeta Potential}

The particle size, polydispersity index (PDI) and zeta potential of liposomes formulations were detected by dynamic and electrophoretic light scattering using a NICOMPTM 380 submicron particle analyzer (PSS Nicomp, Santa Babara, CA, USA). The instruments were equipped with a He-Ne laser at a wavelength of $632.8 \mathrm{~nm}$ at $25^{\circ} \mathrm{C}$. Measurements were taken at a fixed scattering angle of $90^{\circ}$.

\subsubsection{Morphology of Liposomes}

The morphology of liposome formulations was observed by transmission electron microscopy (TEM, JEOL, Tokyo, Japan). The liposomes were placed on carbon-coated grids, and the surplus was 
removed with filter paper and allowed to air dry at room temperature. Then the liposomes were negatively stained by adding phosphotungstic acid $2 \%(w / v)$ solution onto the grids. The samples were air dried overnight at room temperature before measurement by TEM.

\subsubsection{Determination of the Encapsulation Efficiency}

Sephadex G-50 column was used to separate the DiR labeled liposomes and unentrapped DiR. The DiR entrapped in liposomes (Wenc) was dissolved with methanol and detected by a microplate reader fluorescence spectrophotometer (Bio-Rad Laboratories, Inc., Hercules, CA, USA) at 750/790 nm. The total amount of DiR (Wtol) of the liposomes was dissolved with methanol and detected. The encapsulation efficiency (EE) of the liposome formulations was calculated using the formula: $\mathrm{EE}=($ Wenc $) /($ Wtol $) \times 100 \%$.

\subsection{Determination of the Fixed Aqueous Layer Thickness (FALT)}

A classic method based on the Gouy-Chapman theory was used to measure the FALTs of CLip, PEG-Lip and PSA-Lip [34,35]. 10, 50, 100, 150, and $200 \mathrm{mM} \mathrm{NaCl}$ solutions were prepared. To avoid the influence of trace divalent ions on the determination, $0.1 \mathrm{mM}$ EDTA-2Na was added.

$$
\begin{gathered}
\kappa=\sqrt{C} / 0.3 \\
\operatorname{Ln} \psi(L)=\operatorname{Ln} A-k L
\end{gathered}
$$

where $\kappa$ is the Debye-Hückel parameter and $A$ is regarded as a constant and $C$ is the molar concentration of $\mathrm{NaCl}$. The zeta potentials of the varying $\mathrm{NaCl}$ concentrations are measured and plotted against $\kappa$, the slope $L$ gives the FALT in nm units.

\subsection{Pharmacokinetics of Liposomes in Wistar Rats}

\subsubsection{Pharmacokinetics of Liposomes after Single Injection}

Male wistar rats were randomly divided into three groups $(n=3)$. For the pharmacokinetics study involving a single injection, DiR labeled CLip, PEG-Lip and PSA-Lip were intravenously with a phospholipid concentration of $5 \mu \mathrm{mol} / \mathrm{kg}$ via the tail vein under ether anesthesia. Blood samples were obtained from orbital venous plexus and placed into heparinized centrifuge tubes at 1,5,15, and $30 \mathrm{~min}$, $1,4,8,12$, and $24 \mathrm{~h}$ after the injection. Plasma was isolated from blood samples by centrifugation $(2264 \times g, 10 \mathrm{~min})$. The plasma was precipitated with methanol and centrifuged at $11,180 \times g$ for $10 \mathrm{~min}$. Then the obtained supernatant was added into 96-well plate and detected with microplate reader fluorescence spectrophotometer with excitation/emission wavelengths at 750/790 nm, respectively. DAS.2 software was used to calculate the pharmacokinetics parameters.

\subsubsection{Pharmacokinetics and Biodistribution of Liposomes after Repeated Injections}

To investigate the biodistribution and pharmacokinetics involving the repeated injection, the detailed protocols descript in Table 1. The PSA-Lip and PEG-Lip were intravenously injected via the tail vein under ether anesthesia as the first injection. Control group received an injection of $5 \%$ Glu. 7 days later, the blood samples were obtained $0.5 \mathrm{~h}$ before the repeated administrations. DiR labeled PSA-Lip and PEG-Lip were injected intravenously at a dose of $5 \mu \mathrm{mol}$ phospholipid $/ \mathrm{kg}$, the blood samples were got from the orbital venous plexus at 1, 5, 15, and $30 \mathrm{~min}, 1,2$, and $4 \mathrm{~h}$ after the second administration. After the last time point, the rats were sacrificed and livers and spleens were excised. The collected blood samples were processed using the same procedure described in Section 2.7.1. The tissue were homogenized using a Tissue Tearor and precipitated with methanol and centrifuged at 11,180 $\times \mathrm{g}$ for $10 \mathrm{~min}$. Then the obtained supernatant was added into 96-well plate and detected with microplate reader fluorescence spectrophotometer with excitation/emission wavelengths at $750 / 790 \mathrm{~nm}$. 
Table 1. The injection scheme of PSA-Lip and PEG-Lip in wistar rats.

\begin{tabular}{ccl}
\hline Group & $\begin{array}{c}\text { First Injection } \\
\text { (Blank Liposomes) }\end{array}$ & $\begin{array}{c}\text { Second Injection } \\
\text { (DiR Labeled Liposomes) }\end{array}$ \\
\hline A & $5 \%$ Glu & PSA-Lip $(5 \mu \mathrm{mol}$ phospholipid $/ \mathrm{kg})$ \\
B & $5 \%$ Glu & PEG-Lip $(5 \mu \mathrm{mol}$ phospholipid $/ \mathrm{kg})$ \\
C & PSA-Lip $(0.1 \mu \mathrm{mol}$ phospholipid $/ \mathrm{kg})$ & PSA-Lip $(5 \mu \mathrm{mol}$ phospholipid $/ \mathrm{kg})$ \\
D & PSA-Lip $(5 \mu \mathrm{mol}$ phospholipid $/ \mathrm{kg})$ & PSA-Lip $(5 \mu \mathrm{mol}$ phospholipid $/ \mathrm{kg})$ \\
E & PEG-Lip $(0.1 \mu \mathrm{mol}$ phospholipid $/ \mathrm{kg})$ & PEG-Lip $(5 \mu \mathrm{mol}$ phospholipid $/ \mathrm{kg})$ \\
F & PEG-Lip $(5 \mu \mathrm{mol}$ phospholipid $/ \mathrm{kg})$ & PEG-Lip $(5 \mu \mathrm{mol}$ phospholipid $/ \mathrm{kg})$ \\
\hline
\end{tabular}

\subsection{Pharmacokinetics of Liposomes in Beagle Dogs}

Male beagle dogs were randomly divided into 4 groups $(n=3)$. The test protocols were show in Table 2. The beagle dogs were intravenously injected in right forelimb with PSA-Lip and PEG-Lip at a dose of $5 \mu \mathrm{mol}$ phospholipid $/ \mathrm{kg}$. Control group received an injection of $5 \%$ Glu. 7 days later, the blood samples were obtained $0.5 \mathrm{~h}$ before the repeated administrations. DiR labeled PEG-Lip and PSA-Lip were injected intravenously at a dose of $5 \mu \mathrm{mol}$ phospholipid $/ \mathrm{kg}$ for the second injection. At various time points of 1, 5, 15, and $30 \mathrm{~min}, 1,2$, and $4 \mathrm{~h}$ after injection, $2 \mathrm{~mL}$ of blood from the left forelimb vein was placed in a heparinized tube, and processed using the same procedure described in Section 2.7.1.

Table 2. The injection scheme of PSA-Lip and PEG-Lip in beagle dogs.

\begin{tabular}{ccc}
\hline Group & $\begin{array}{c}\text { First Injection } \\
\text { (Blank Liposomes) }\end{array}$ & $\begin{array}{c}\text { Second Injection } \\
\text { (DiR Labeled Liposomes) }\end{array}$ \\
\hline I & $5 \%$ Glu & PSA-Lip $(5 \mu \mathrm{mol}$ phospholipid $/ \mathrm{kg})$ \\
II & $5 \%$ Glu & PEG-Lip $(5 \mu \mathrm{mol}$ phospholipid $/ \mathrm{kg})$ \\
III & PSA-Lip $(5 \mu \mathrm{mol}$ phospholipid $/ \mathrm{kg})$ & PSA-Lip $(5 \mu \mathrm{mol}$ phospholipid $/ \mathrm{kg})$ \\
IV & PEG-Lip $(5 \mu \mathrm{mol}$ phospholipid $/ \mathrm{kg})$ & PEG-Lip $(5 \mu \mathrm{mol}$ phospholipid $/ \mathrm{kg})$ \\
\hline
\end{tabular}

\subsection{Quantification of IgM}

PSA-BS18 and $\mathrm{mPEG}_{2000}$-DSPE $(0.2 \mathrm{mM})$ were added into a 96-well plate, and the coated plate was dried at room temperature. Tris-buffer containing $1 \%$ BSA solution was added to block the wells. Then the wells were washed three times with a Tris buffered saline solution containing $0.05 \%$ CHAPS. $100-\mu \mathrm{L}$ of diluted serum samples of rats and beagle dogs were then added to the wells respectively and incubated for $90 \mathrm{~min}$, and then washed 5 times as described above. Horseradish peroxidase -conjugated rabbit anti-rat/dog antibody was added into each well $(100 \mu \mathrm{L} /$ well $)$ at a concentration of $1 \mu \mathrm{g} / \mathrm{mL}$. After $60 \mathrm{~min}$ incubation at room temperature, the wells were washed 5 times. $1 \mathrm{mg} / \mathrm{mL}$ of o-phenylene diamine $(100 \mu \mathrm{L} /$ well $)$ was added into the wells and incubated for $15 \mathrm{~min} .2 \mathrm{M} \mathrm{H}_{2} \mathrm{SO}_{4}(100 \mu \mathrm{L} /$ well $)$ was added to terminate the reaction. The absorbance was detected at $490 \mathrm{~nm}$ by microplate reader fluorescence spectrophotometer.

\subsection{Statistical Analysis}

All data were expressed as the mean \pm S.D. Statistical comparisons were calculated by Students $t$-test or Tukey's test with SPSS 17.0 software. $p<0.05$ is considered as statistically significant. $p<0.01$ is considered as statistically extremely significant.

\section{Results and Discussion}

\subsection{The Characteristics of the Prepared Liposomes}

The physicochemical property of the prepared liposome were presented in Table 3. The mean particle size of the liposome formulations was $90-110 \mathrm{~nm}$. The zeta potentials varied from -10 to $-20 \mathrm{mV}$. The morphology of liposomes was observed using TEM. As shown in Figure 1, the prepared 
liposomes were in near spherical shape. The encapsulation efficiency of the DiR in the labeled liposomes were all above 95\%, which was in agreement with the previous study [30,31] from our group.

Table 3. The characteristics of the prepared liposomes.

\begin{tabular}{cccccc}
\hline Formulation & Particle Size $(\mathbf{n m})$ & Polydispersity & Zeta Potential $(\mathbf{m V})$ & EE $(\%)$ & FALT $(\mathbf{n m})$ \\
\hline Clip & $128.4 \pm 3.4$ & $0.154 \pm 0.012$ & $-12.1 \pm 3.0$ & $95.2 \pm 1.6$ & $0.28 \pm 0.04$ \\
PSA-Lip & $125.6 \pm 8.3$ & $0.164 \pm 0.021$ & $-18.6 \pm 5.1$ & $96.3 \pm 1.4$ & $0.83 \pm 0.05$ \\
PEG-Lip & $111.3 \pm 5.6$ & $0.132 \pm 0.011$ & $-16.7 \pm 2.6$ & $96.1 \pm 2.3$ & $2.61 \pm 0.08$ \\
\hline
\end{tabular}
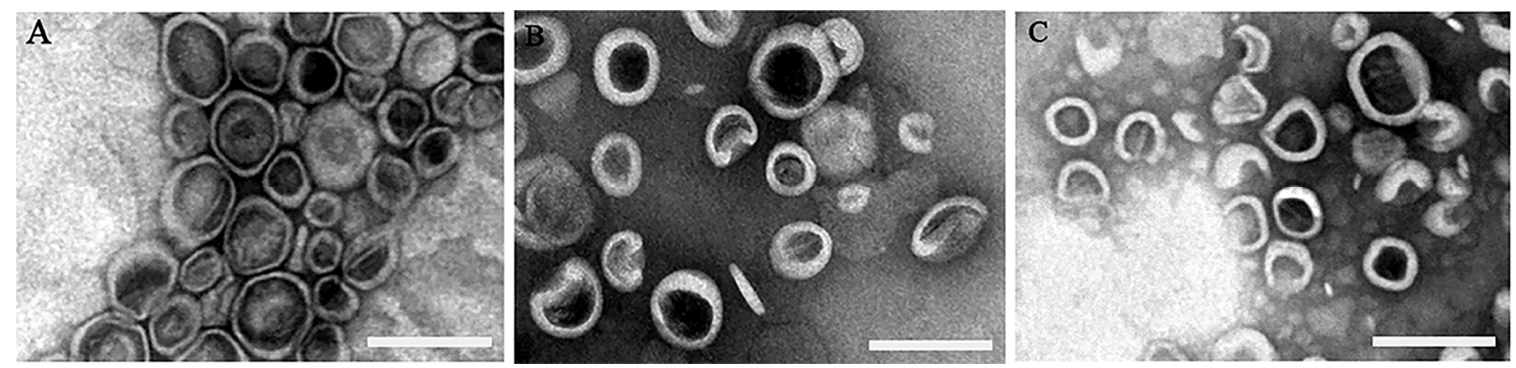

Figure 1. The TEM images of (A) Clip, (B) PSA-Lip, and (C) PEG-lip. Scale bars represented $200 \mathrm{~nm}$.

\subsection{The Comparison of FALT around Liposomes}

FALT is considered to influence the biochemical property of the polymer-modified liposomes. Shimada et al. [36] established a method to measure the FALT around liposomes. The results showed that as FALT on the liposomes increased, macrophage uptake decreased, which led to a prolonged circulation time in blood. Thus, FALT was used as a quantitative measure of liposomes for avoiding opsonization and uptake by the MPS.

From Equations (1) and (2) in Section 2.6: the FALTs of the Clip, PSA-Lip and PEG-Lip were determined to be $0.28,0.83$ and $2.61 \mathrm{~nm}$, respectively. The FALT of PSA-Lip $(0.83 \mathrm{~nm})$ was thicker than CLip $(0.28 \mathrm{~nm})(p<0.05)$. The increasing FALT of PSA-Lip would led a reduction in uptake by macrophages, resulting in a prolonged blood circulation.

\subsection{Pharmacokinetics of Liposomes after Single Injection in Wistar Rats}

To investigate whether PSA modification could afford the stealthiness in the blood circulation, PSA-Lip were intravenously administered in wistar rats. As shown in Figure 2, the blood clearance rate of PSA-Lip was significantly slower compared to that of CLip. Table 4 presented the pharmacokinetic parameters. The area under the blood concentration-time curve (AUC) of PSA-Lip was significantly higher than Clip $(p<0.01)$. The clearance $(\mathrm{CLz})$ value of PSA-Lip $\left(0.085 \pm 0.008 \mathrm{~L} \cdot \mathrm{kg}^{-1} \cdot \mathrm{h}^{-1}\right) \mathrm{was}$ significantly lower $(p<0.01)$ than Clip $\left(0.512 \pm 0.141 \mathrm{~L} \cdot \mathrm{kg}^{-1} \cdot \mathrm{h}^{-1}\right)$. In addition, the mean residence time (MRT) of PSA-Lip $(9.23 \pm 0.46 \mathrm{~h})$ also increased significantly $(p<0.01)$ in comparison to that of Clip $(5.12 \pm 1.01 \mathrm{~h})$. The results showed that PSA-Lip could afford extended blood circulation time in vivo. The immunological properties of $\alpha$-2,8-linked PSA were reported to be consistent with those in mammals [24]. Therefore, PSA can be used as an endogenous immune camouflage material to protect the modified liposomes from recognizing by the immune system. Meanwhile, numerous studies $[30,31,37,38]$ have shown that PSA can prolong the circulation time of modified drugs/carriers in vivo. Among them, the determination of PSA's fixed hydration layer thickness (FALT) corroborates the hydration cloud theory proposed by Bader [34], and the presence of FALT can help PSA modified drugs/carriers escape the recognition of opsonins in the blood, decrease MPS uptake and prolong residence time in the body. 


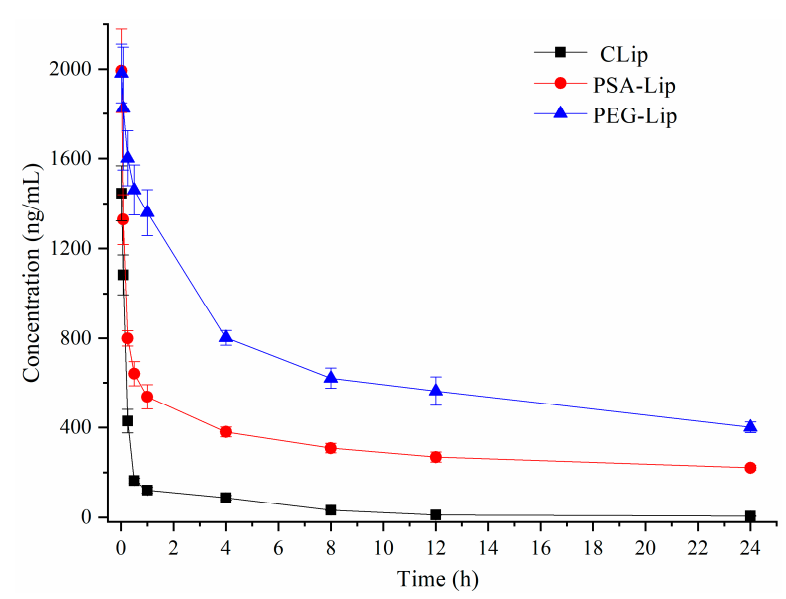

Figure 2. The blood clearance of various DiR labeled liposomes after intravenous administration in wistar rats at a dose of $5 \mu \mathrm{mol}$ phospholipids $/ \mathrm{kg}$. Data were shown as mean \pm S.D., $n=3$.

Table 4. Pharmacokinetic parameters of various DiR labeled liposomes after single intravenous administration in wistar rats $(n=3)$.

\begin{tabular}{cccc}
\hline Parameter & CLip & PSA-Lip & PEG-Lip \\
\hline Cmax $\left(\mu \mathrm{g} \cdot \mathrm{L}^{-1}\right)$ & $1146.3 \pm 233.2$ & $1992.3 \pm 125.6$ & $1996.3 \pm 114.3$ \\
AUC $(0-t)\left(\mu \mathrm{g} \cdot \mathrm{L}^{-1} \cdot \mathrm{h}\right)$ & $2223.3 \pm 125.6$ & $7213.8 \pm 100.27$ & $15781.4 \pm 336.28$ \\
MRT $(0-t)(\mathrm{h})$ & $5.12 \pm 1.01$ & $9.23 \pm 0.46$ & $12.32 \pm 0.65$ \\
CLz $\left(\mathrm{L} \cdot \mathrm{kg}^{-1} \cdot \mathrm{h}^{-1}\right)$ & $0.512 \pm 0.141$ & $0.085 \pm 0.008$ & $0.017 \pm 0.004$ \\
\hline
\end{tabular}

\subsection{Pharmacokinetics and Bio-Distribution of Various Liposomes after the Repeated Injections in Wistar Rats}

Ishida et al. [19] pointed out that when PEGylated liposomes were intravenously injected at a dose of $0.001-0.1 \mu \mathrm{mol}$ phospholipid $/ \mathrm{kg}$ as the first injection, the ABC phenomenon clearly occurred upon repeated injections. Furthermore, five to seven day intervals between PEGylated liposome injections induced much more pronounced ABC phenomenon [39-43].

To explore whether the ABC phenomenon would be induced by the PSA modification in wistar rats, blank liposomes were intravenously administered as the first injection, and the corresponding DiR labeled liposomes ( $5 \mu \mathrm{mol}$ phospholipid $/ \mathrm{kg}$ ) were administered intravenously seven days later. As illustrated in Figure 3A, after repeated injections, PEGylated liposomes were rapidly cleared $(p<0.01)$. The blood clearance of pre-injection with PEG-Lip at the dose of $0.1 \mu \mathrm{mol}$ phospholipid $/ \mathrm{kg}$ was higher than that of $5 \mu \mathrm{mol}$ phospholipid $/ \mathrm{kg}$. The accumulation in liver and spleen increased significantly $(p<0.01)$ after the repeated injections (Figure 3B). The results indicated that PEGylated liposomes caused an obvious ABC phenomenon. As for PSA-Lip, the blood clearance curves were very similar between the single dose and second injection both at the dose of 0.1 and $5 \mu \mathrm{mol}$ phospholipid $/ \mathrm{kg}$ (Figure 4A). Hepatic and splenic aggregation also did not increase significantly $(p>0.05)$ in comparison with the control group (Figure 4B). The results suggested that PSA-Lip did not induce the ABC phenomenon after repeated injections.

According to reports, the $A B C$ index can be used as an effective parameter to evaluate the degree of $A B C$ phenomenon. The $A B C$ index = AUC of the second dose/AUC of the first dose. A higher $A B C$ index means a weaker induction of the $A B C$ phenomenon [44]. Interestingly, a study from Zhao et al. [45] has implied that $A B C$ phenomenon is suitable for evaluation by the $A B C$ index (0-30 min). In the present study, the $A B C$ index (0-30 min) of Group C and Group D were $1.01 \pm 0.06$ and $0.98 \pm 0.08$ respectively, while Group E and Group F was $0.71 \pm 0.13$ and $0.23 \pm 0.04$, respectively. These results also demonstrated that PSA-Lip did not induce the ABC phenomenon. 


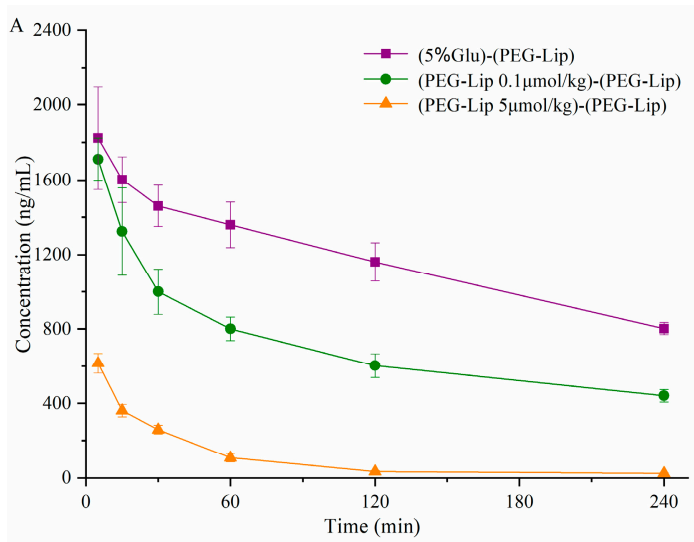

(A)

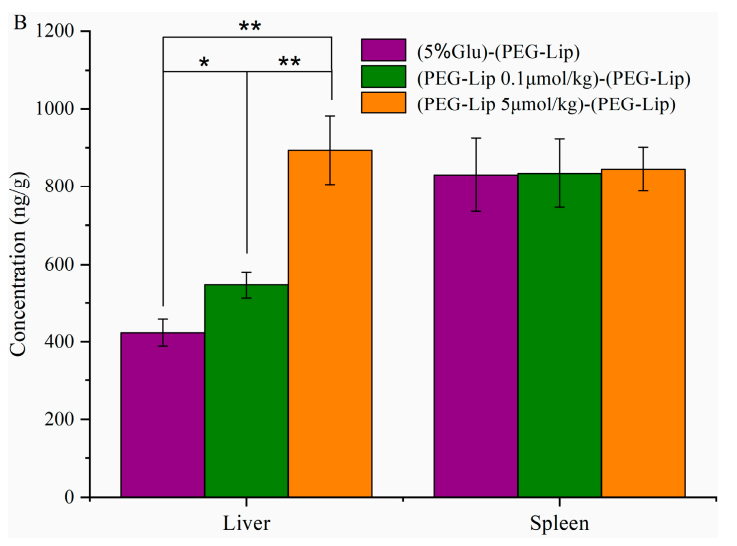

(B)

Figure 3. (A) Pharmacokinetic profile in wistar rats after the repeated injection of PEG-Lip (0-4 h). (B) Hepatic and splenic accumulation $(4 \mathrm{~h})$ in rats after second injection of PEG-Lip. Data were shown as mean \pm S.D., $n=3$. ${ }^{*} p<0.05,{ }^{* *} p<0.01$.

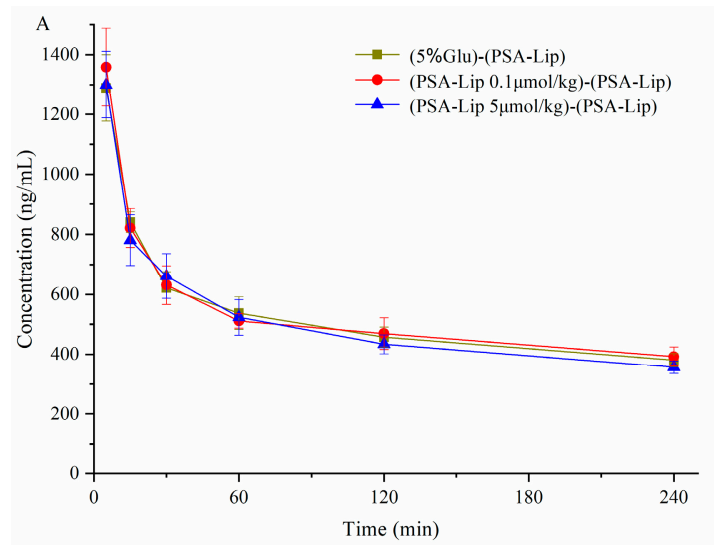

(A)

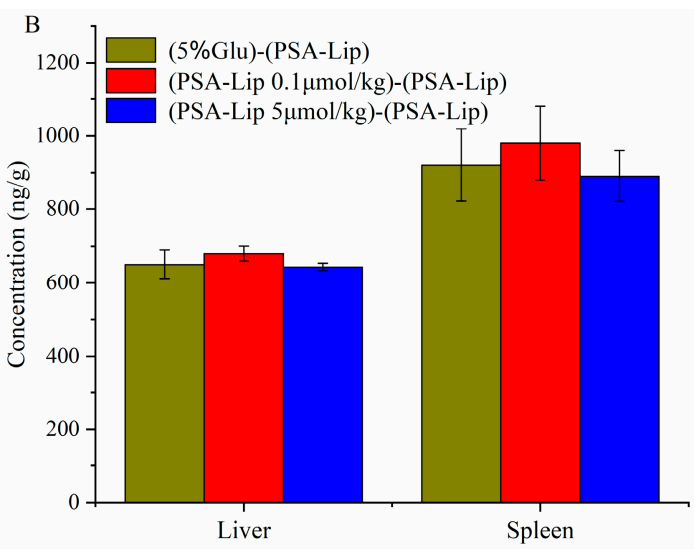

(B)

Figure 4. (A) Pharmacokinetic profile in wistar rats after the repeated injection of PSA-Lip (0-4 h). (B) Hepatic and splenic accumulation $(4 \mathrm{~h})$ in rats after second injection of PSA-Lip. Data were shown as mean \pm S.D., $n=3$.

\subsection{Pharmacokinetics of Various Liposomes after the Injections in Beagle Dogs}

Different animal models have been used to study the $\mathrm{ABC}$ phenomenon, such as mice, rats, rabbits, beagle dogs and so on [13]. Recently, Suzuki and coworkers [46] revealed that beagle dogs were more sensitive to the immune response than other animals models. Under the same degree of stimulation, $A B C$ phenomenon is more likely to be induced in beagle dogs. The dogs could produce the severe $\mathrm{ABC}$ phenomenon when administrated with blank PEGylated liposomes at a higher phospholipid dose of $16.7 \mu \mathrm{mol}$ phospholipids $/ \mathrm{kg}$, while in the rats were pretreated with a high dose $(\geq 5 \mu \mathrm{mol}$ phospholipids $/ \mathrm{kg}$ ), the ABC phenomenon was weakened [19,47]. Hence, difference between species should be considered in the study of long-circulation formulations. In the present study, the beagle dog was also selected as animal model to study the ABC phenomenon of the repeated injections of PSA-Lip, which could investigate the immunogenicity of PSA-Lip comprehensively.

As shown in Figure 5, the DiR of the plasma decreased significantly after repeated injections of PEG-Lip in beagle dogs, and the ABC index (0-30 min) was $0.09 \pm 0.02$. No obvious change between the single and repeated injections of PSA-Lip was observed, the ABC index (0-30 min) was 1.04 \pm 0.05 . The results revealed that the $\mathrm{ABC}$ phenomenon was not triggered by PSA-Lip after repeated injections in the beagle dogs. 


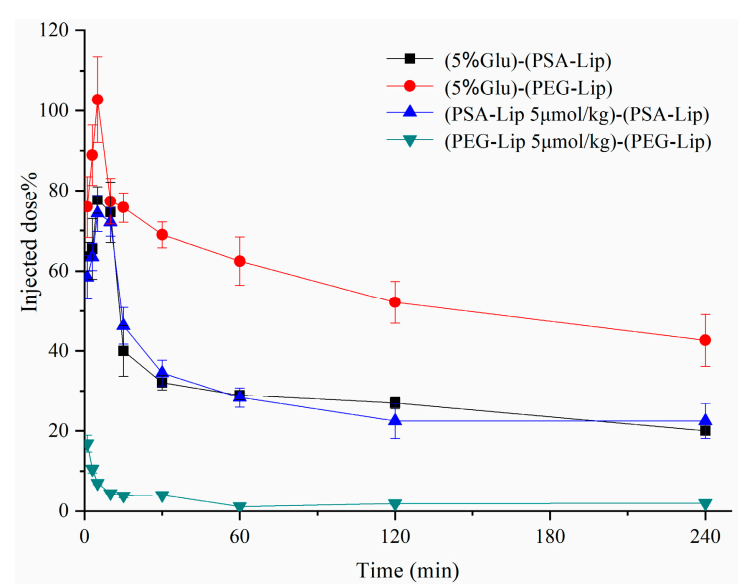

Figure 5. Pharmacokinetic profile $(0-4 \mathrm{~h})$ in beagle dogs after intravenous single and repeated injections of DiR labeled liposomes. Data were shown as mean \pm S.D., $n=3$.

\subsection{Determination of the Production of Anti-PEG (PSA) IgM}

The IgM antibody was produced in the first injection as an immune response, which would stimulate the complement system and resulted in uptake by Kupffer cell endocytosis following the second injection of liposomes [48]. As illustrated in Figure 6, the first injection of PEG-Lip significantly produced IgM antibody (compared to control, $p<0.01$ ). In contrast, the first injection of PSA-Lip did not induce the IgM in the serum, and would not bound on the second injection of PSA-Lip.

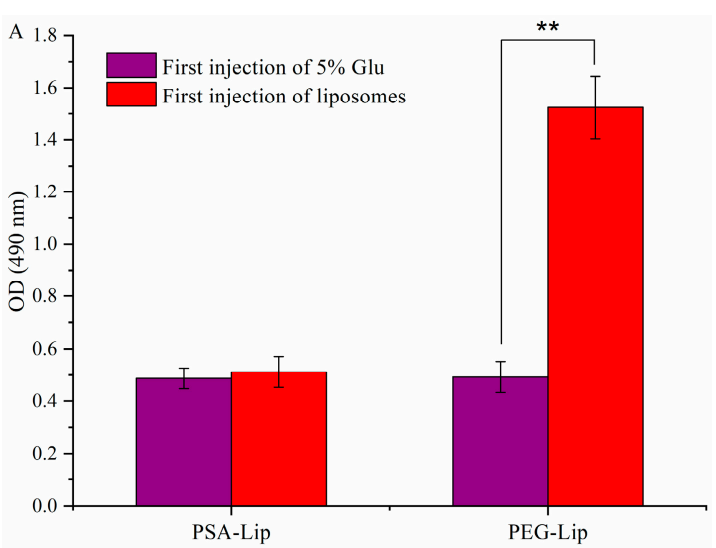

(A)

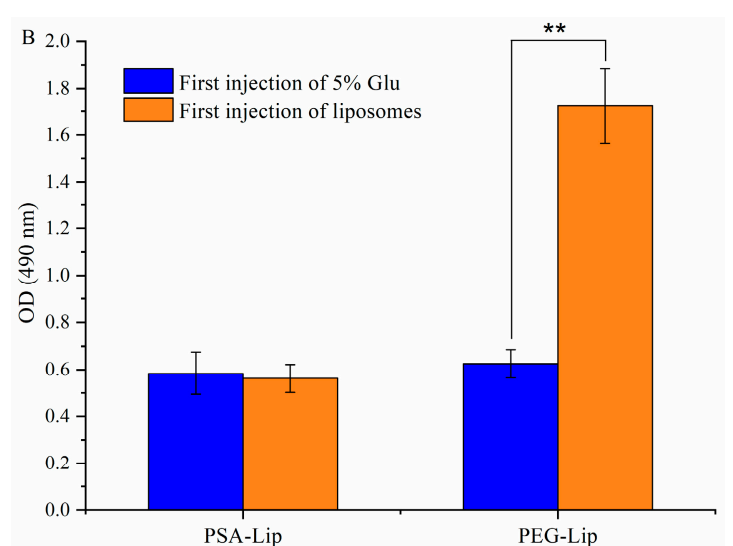

(B)

Figure 6. Anti-PEG (PSA) IgM production induced by the initial injection (A) in wistar rats and (B) in beagle dogs. Data were shown as mean \pm S.D., $n=3$. ${ }^{* *} p<0.01$.

A previous study [13] reported that PEG on the PEGylated liposomes acted as a TI-2 antigen and the repeating subunit of PEG may be an immunogenic epitope. This epitope may be a binding site for the derived anti-PEG IgM, which induced fast blood clearance in vivo following the second injection. The results in our study showed that the first injection of PSA-Lip did not induce IgM production, resulting in no combination with the second injection of PSA-Lip. PSA is a linear polymer connected by Neu5Ac with $\alpha-2,8$ ketone glycoside bonds. It is an endogenous substance, which is biocompatible and non-immunogenic [20,49,50]. Fernandes et al. [21] also proposed that the immunogenicity of PSA-conjugated asparaginase did not increase against naive asparaginase. These findings were consistent with the above results.

In this study, two animal models of wistar rat and beagle dog are used to investigate the ABC phenomenon of PSA modified liposomes for the repeated injections. The results showed that the first injection of PSA-modified liposomes in wistar rats or beagle dogs did not cause changes in the pharmacokinetic behavior of the second injection and did not produce $\mathrm{ABC}$ phenomenon. On the 
one hand, PSA is no-immunogenic and does not cause an immune response in vivo [51]; on the other hand, PSA can inhibit the body's immune response, and the modified liposome does not induce the production of anti-PSA-IgM, which plays an immune camouflage role. Therefore, no ABC phenomenon was induced.

Our research group has devoted the effect to overcoming the $A B C$ phenomenon caused by PEGylated formulations in recent years. Studied pointed out that not only PEGylated liposomes [14] could induce the ABC phenomenon, but also repeated injections of PEGylated solid lipid nanoparticles [45], PEGylated emulsions [52] and PEGylated micelles [53] could trigger ABC phenomenon. A variety of strategies have been proposed to address this dilemma, such as, amelioration of dosing regimen [52], alteration of linkages of PEG derivatives and molecular weights of PEG [54], but they were still unsatisfactory in eliminating the $\mathrm{ABC}$ phenomenon. As an endogenous substance, PSA is non-immunogenicity and can escape immune system surveillance through immune camouflage. In the previous work, we modified PSA derivatives on the surface of liposomal chemotherapy drugs for anti-tumor therapy, and the results showed that PSA modification exhibited an extended circulation time and excellent anti-tumor efficacy [30,31]. To further verify its application prospects in drug delivery systems, the ABC phenomenon of PSA modified liposomes in beagle dogs and wistar rats was conducted. The results of this study showed that repeated injections of PSA would not induce the $\mathrm{ABC}$ phenomenon in both beagle dogs and wistar rats, which presents a promising approach for constructing drug delivery system.

\section{Conclusions}

In the present study, PSA was selected to modified liposomes and a systematic study was conducted. Because of the hydrophilic and non-immunogenic properties, PSA can be used as a modification material to extend the circulation time in drug delivery system. And the $\mathrm{ABC}$ phenomenon and IgM antibody production was not induced after repeated injections. Because of its inherent biodegradability and immunogenicity, PSA represents a good option for drug delivery in clinical treatments that require prolonged intravenous therapy.

Author Contributions: Methodology, X.H. and M.L.; data curation, X.H. and T.Z.; writing—original draft preparation, X.H. and T.Z.; writing-review and editing, Y.S.; project administration, X.L.; funding acquisition, Y.D. All authors have read and agreed to the published version of the manuscript.

Funding: This research was funded by the National Natural Science Foundation of China. (NO.81973271 and 81903808).

Conflicts of Interest: The authors declare no conflict of interest.

\section{References}

1. Howard, M.D.; Jay, M.; Dziublal, T.D.; Lu, X. PEGylation of nanocarrier drug delivery systems: State of the art. J. Biomed. Nanotechnol. 2008, 4, 133-148. [CrossRef]

2. Hussain, Z.; Khan, S.; Imran, M.; Sohail, M.; Shah, S.W.A.; de Matas, M. PEGylation: A promising strategy to overcome challenges to cancer-targeted nanomedicines: A review of challenges to clinical transition and promising resolution. Drug Deliv. Transl. Res. 2019, 9, 721-734. [CrossRef] [PubMed]

3. Allen, T.M.; Hansen, C.; Martin, F.; Redemann, C.; Yau-Young, A. Liposomes containing synthetic lipid derivatives of poly(ethylene glycol) show prolonged circulation half-lives in vivo. Biochim. Biophys. Acta 1991, 1066, 29-36. [CrossRef]

4. Van Vlerken, L.E.; Vyas, T.K.; Amiji, M.M. Poly(ethylene glycol)-modified nanocarriers for tumor-targeted and intracellular delivery. Pharm. Res. 2007, 24, 1405-1414. [CrossRef]

5. Knop, K.; Hoogenboom, R.; Fischer, D.; Schubert, U.S. Poly(ethylene glycol) in drug delivery: Pros and cons as well as potential alternatives. Angew. Chem. Int. Ed. 2010, 49, 6288-6308. [CrossRef]

6. Allen, T.M.; Cullis, P.R. Liposomal drug delivery systems: From concept to clinical applications. Adv. Drug Deliv. Rev. 2013, 65, 36-48. [CrossRef] 
7. Park, J.-H.; Cho, H.-J.; Yoon, H.Y.; Yoon, I.-S.; Ko, S.-H.; Shim, J.-S.; Cho, J.-H.; Park, J.H.; Kim, K.; Kwon, I.C.; et al. Hyaluronic acid derivative-coated nanohybrid liposomes for cancer imaging and drug delivery. J. Control. Release 2014, 174, 98-108. [CrossRef]

8. Ellens, H.; Bentz, J.; Szoka, F.C. PH-induced destabilization of phosphatidylethanolamine-containing liposomes: Role of bilayer contact. Biochemistry 1984, 23, 1532-1538. [CrossRef]

9. Ishida, T.; Harashima, H.; Kiwada, H. Liposome clearance. Biosci. Rep. 2002, 22, 197-224. [CrossRef]

10. Maeda, H.; Wu, J.; Sawa, T.; Matsumura, Y.; Hori, K. Tumor vascular permeability and the EPR effect in macromolecular therapeutics: A review. J. Control. Release 2000, 65, 271-284. [CrossRef]

11. Manson, J.; Kumar, D.; Meenan, B.J.; Dixon, D. Polyethylene glycol functionalized gold nanoparticles: The influence of capping density on stability in various media. Gold Bull 2011, 44, 99-105. [CrossRef]

12. Fishburn, C.S. The pharmacology of PEGylation: Balancing PD with PK to generate novel therapeutics. J. Pharm. Sci. 2008, 97, 4167-4183. [CrossRef] [PubMed]

13. Abu Lila, A.S.; Kiwada, H.; Ishida, T. The accelerated blood clearance (ABC) phenomenon: Clinical challenge and approaches to manage. J. Control. Release 2013, 172, 38-47. [CrossRef]

14. Dams, E.T.M.; Laverman, P.; Oyen, W.J.G.; Storm, G.; Scherphof, G.L.; van der Meer, J.W.M.; Corstens, F.H.M.; Boerman, O.C. Accelerated blood clearance and altered biodistribution of repeated injections of sterically stabilized liposomes. J. Pharmacol. Exp. Ther. 2000, 292, 1071-1079. [PubMed]

15. Mohamed, M.; Abu Lila, A.S.; Shimizu, T.; Alaaeldin, E.; Hussein, A.; Sarhan, H.A.; Szebeni, J.; Ishida, T. PEGylated liposomes: Immunological responses. Sci. Technol. Adv. Mater. 2019, 20, 710-724. [CrossRef] [PubMed]

16. Suzuki, T.; Ichihara, M.; Hyodo, K.; Yamamoto, E.; Ishida, T.; Kiwada, H.; Kikuchi, H.; Ishihara, H. Influence of dose and animal species on accelerated blood clearance of PEGylated liposomal doxorubicin. Int. J. Pharm. 2014, 476, 205-212. [CrossRef]

17. Kozma, G.T.; Mészáros, T.; Vashegyi, I.; Fülöp, T.; Örfi, E.; Dézsi, L.; Rosivall, L.; Bavli, Y.; Urbanics, R.; Mollnes, T.E.; et al. Pseudo-anaphylaxis to polyethylene glycol (PEG)-coated liposomes: Roles of anti-PEG IgM and complement activation in a porcine model of human infusion reactions. ACS Nano 2019, 13, 9315-9324. [CrossRef]

18. Ishida, T.; Ichikawa, T.; Ichihara, M.; Sadzuka, Y.; Kiwada, H. Effect of the physicochemical properties of initially injected liposomes on the clearance of subsequently injected PEGylated liposomes in mice. J. Control. Release 2004, 95, 403-412. [CrossRef]

19. Ishida, T.; Harada, M.; Wang, X.Y.; Ichihara, M.; Irimura, K.; Kiwada, H. Accelerated blood clearance of PEGylated liposomes following preceding liposome injection: Effects of lipid dose and PEG surface-density and chain length of the first-dose liposomes. J. Control. Release 2005, 105, 305-317. [CrossRef]

20. Gregoriadis, G.; Fernandes, A.; Mital, M.; McCormack, B. Polysialic acids: Potential in improving the stability and pharmacokinetics of proteins and other therapeutics. Cell. Mol. Life Sci. 2000, 57, 1964-1969. [CrossRef]

21. Fernandes, A.I.; Gregoriadis, G. The effect of polysialylation on the immunogenicity and antigenicity of asparaginase: Implication in its pharmacokinetics. Int. J. Pharm. 2001, 217, 215-224. [CrossRef]

22. Kiss, J.Z.; Rougon, G. Cell biology of polysialic acid. Curr. Opin. Neurobiol. 1997, 7, 640-646. [CrossRef]

23. Yang, P.; Major, D.; Rutishauser, U. Role of charge and hydration in effects of polysialic acid on molecular interactions on and between cell membranes. J. Biol. Chem. 1994, 269, 23039-23044. [PubMed]

24. Muhlenhoff, M.; Eckhardt, M.; Gerardy-Schahn, R. Polysialic acid: Three-dimensional structure, biosynthesis and function. Curr. Opin. Struct. Biol. 1998, 8, 558-564. [CrossRef]

25. Ram, S.; Sharma, A.K.; Simpson, S.D.; Gulati, S.; McQuillen, D.P.; Pangburn, M.K.; Rice, P.A. A novel sialic acid binding site on factor $\mathrm{H}$ mediates serum resistance of sialylated Neisseria gonorrhoeae. J. Exp. Med. 1998, 187, 743-752. [CrossRef]

26. Gunawan, J.; Simard, D.; Gilbert, M.; Lovering, A.L.; Wakarchuk, W.W.; Tanner, M.E.; Strynadka, N.C.J. Structural and mechanistic analysis of sialic acid synthase NeuB from Neisseria meningitidis in complex with $\mathrm{Mn}^{2+}$, phosphoenolpyruvate, and N-acetylmannosaminitol. J. Biol. Chem. 2005, 280, 3555-3563. [CrossRef]

27. Bader, R.A.; Wardwell, P.R. Polysialic acid: Overcoming the hurdles of drug delivery. Ther. Deliv. 2014, 5, 235-237. [CrossRef]

28. Kou, Y.M.; Feng, R.; Chen, J.P.; Duan, L.L.; Wang, S.Y.; Hu, Y.W.; Zhang, N.; Wang, T.Y.; Deng, Y.H.; Song, Y.Z. Development of a nattokinase-polysialic acid complex for advanced tumor treatment. Eur. J. Pharm. Sci. 2020, 145, 13. [CrossRef] 
29. Zhang, T.; Zhou, S.; Hu, L.; Peng, B.; Liu, Y.; Luo, X.; Liu, X.; Song, Y.; Deng, Y. Polysialic acid-polyethylene glycol conjugate-modified liposomes as a targeted drug delivery system for epirubicin to enhance anticancer efficiency. Drug Deliv. Transl Res. 2018, 8, 602-616. [CrossRef]

30. Luo, X.; Liu, M.; Hu, L.; Qiu, Q.; Liu, X.; Li, C.; Lu, M.; Liu, Y.; Zhang, T.; Zhou, S.; et al. Targeted delivery of pixantrone to neutrophils by poly(sialic acid)-p-octadecylamine conjugate modified liposomes with improved antitumor activity. Int. J. Pharm. 2018, 547, 315-329. [CrossRef]

31. Zhang, T.; Zhou, S.; Hu, L.; Peng, B.; Liu, Y.; Luo, X.; Song, Y.; Liu, X.; Deng, Y. Polysialic acid-modifying liposomes for efficient delivery of epirubicin, in-vitro characterization and in-vivo evaluation. Int. J. Pharm. 2016, 515, 449-459. [CrossRef] [PubMed]

32. Kilkenny, C.; Browne, W.J.; Cuthill, I.C.; Emerson, M.; Altman, D.G. Improving bioscience research reporting: The ARRIVE guidelines for reporting animal research. PLoS Biol. 2010, 8. [CrossRef] [PubMed]

33. Epstein, H.; Gutman, D.; Cohen-Sela, E.; Haber, E.; Elmalak, O.; Koroukhov, N.; Danenberg, H.D.; Golomb, G. Preparation of alendronate liposomes for enhanced stability and bioactivity: In vitro and in vivo characterization. AAPS J. 2008, 10, 505-515. [CrossRef] [PubMed]

34. Wilson, D.R.; Zhang, N.; Silvers, A.L.; Forstner, M.B.; Bader, R.A. Synthesis and evaluation of cyclosporine A-loaded polysialic acid-polycaprolactone micelles for rheumatoid arthritis. Eur. J. Pharm. Sci. 2014, 51, 146-156. [CrossRef] [PubMed]

35. Sadzuka, Y.; Nakade, A.; Hirama, R.; Miyagishima, A.; Nozawa, Y.; Hirota, S.; Sonobe, T. Effects of mixed polyethyleneglycol modification on fixed aqueous layer thickness and antitumor activity of doxorubicin containing liposome. Int. J. Pharm. 2002, 238, 171-180. [CrossRef]

36. Shimada, K.; Miyagishima, A.; Sadzuka, Y.; Nozawa, Y.; Mochizuki, Y.; Ohshima, H.; Hirota, S. Determination of the thickness of the fixed aqueous layer around polyethyleneglycol-coated liposomes. J. Drug Target. 1995, 3, 283-289. [CrossRef]

37. Greco, F.; Arif, I.; Botting, R.; Fante, C.; Quintieri, L.; Clementi, C.; Schiavon, O.; Pasut, G. Polysialic acid as a drug carrier: Evaluation of a new polysialic acid-epirubicin conjugate and its comparison against established drug carriers. Polym. Chem. 2013, 4, 1600-1609. [CrossRef]

38. Jain, S.; Hreczuk-Hirst, D.H.; McCormack, B.; Mital, M.; Epenetos, A.; Laing, P.; Gregoriadis, G. Polysialylated insulin: Synthesis, characterization and biological activity in vivo. Biochim. Biophys. Acta 2003, 1622, 42-49. [CrossRef]

39. Ma, Y.; Yang, Q.; Wang, L.; Zhou, X.; Zhao, Y.; Deng, Y. Repeated injections of PEGylated liposomal topotecan induces accelerated blood clearance phenomenon in rats. Eur. J. Pharm. Sci. 2012, 45, 539-545. [CrossRef]

40. Yang, Q.; Ma, Y.; Zhao, Y.; She, Z.; Wang, L.; Li, J.; Wang, C.; Deng, Y. Accelerated drug release and clearance of PEGylated epirubicin liposomes following repeated injections: A new challenge for sequential low-dose chemotherapy. Int. J. Nanomed. 2013, 8, 1257.

41. Wang, C.; Cheng, X.; Sui, Y.; Luo, X.; Jiang, G.; Wang, Y.; Huang, Z.; She, Z.; Deng, Y. A noticeable phenomenon: Thiol terminal PEG enhances the immunogenicity of PEGylated emulsions injected intravenously or subcutaneously into rats. Eur. J. Pharm. BioPharm. 2013, 85, 744-751. [CrossRef] [PubMed]

42. Xu, H.; Wang, K.Q.; Deng, Y.H.; Chen, D.W. Effects of cleavable PEG-cholesterol derivatives on the accelerated blood clearance of PEGylated liposomes. Biomaterials 2010, 31, 4757-4763. [CrossRef] [PubMed]

43. Zhao, Y.; Wang, C.; Wang, L.; Yang, Q.; Tang, W.; She, Z.; Deng, Y. A frustrating problem: Accelerated blood clearance of PEGylated solid lipid nanoparticles following subcutaneous injection in rats. Eur. J. Pharm. BioPharm. 2012, 81, 506-513. [CrossRef] [PubMed]

44. Ishihara, T.; Takeda, M.; Sakamoto, H.; Kimoto, A.; Kobayashi, C.; Takasaki, N.; Yuki, K.; Tanaka, K.-I.; Takenaga, M.; Igarashi, R.; et al. Accelerated blood clearance phenomenon upon repeated injection of PEG-modified PLA-nanoparticles. Pharm. Res. 2009, 26, 2270-2279. [CrossRef]

45. Zhao, Y.; Wang, L.; Yan, M.; Ma, Y.; Zang, G.; She, Z.; Deng, Y. Repeated injection of PEGylated solid lipid nanoparticles induces accelerated blood clearance in mice and beagles. Int. J. Nanomed. 2012, 7, 2891.

46. Suzuki, T.; Ichihara, M.; Hyodo, K.; Yamamoto, E.; Ishida, T.; Kiwada, H.; Ishihara, H.; Kikuchi, H. Accelerated blood clearance of PEGylated liposomes containing doxorubicin upon repeated administration to dogs. Int. J. Pharm. 2012, 436, 636-643. [CrossRef]

47. Ishida, T.; Ichihara, M.; Wang, X.; Yamamoto, K.; Kimura, J.; Majima, E.; Kiwada, H. Injection of PEGylated liposomes in rats elicits PEG-specific IgM, which is responsible for rapid elimination of a second dose of PEGylated liposomes. J. Control. Release 2006, 112, 15-25. [CrossRef] 
48. Wang, X.; Ishida, T.; Kiwada, H. Anti-PEG IgM elicited by injection of liposomes is involved in the enhanced blood clearance of a subsequent dose of PEGylated liposomes. J. Control. Release 2007, 119, 236-244. [CrossRef]

49. Moreno, C.; Lifely, M.R.; Esdaile, J. Immunity and protection of mice against Neisseria meningitidis group B by vaccination, using polysaccharide complexed with outer membrane proteins: A comparison with purified B polysaccharide. Infect. Immun. 1985, 47, 527-533. [CrossRef]

50. Janas, T. Membrane oligo- and polysialic acids. Biochim. Biophys. Acta Biomembr. 2011, 1808, $2923-2932$. [CrossRef]

51. Sato, C.; Kitajima, K. Disialic, oligosialic and polysialic acids: Distribution, functions and related disease. J. Biochem. 2013, 154, 115-136. [CrossRef] [PubMed]

52. Wang, C.; Cheng, X.; Su, Y.; Pei, Y.; Song, Y.; Jiao, J.; Huang, Z.; Ma, Y.; Dong, Y.; Yao, Y.; et al. Accelerated blood clearance phenomenon upon cross-administration of PEGylated nanocarriers in beagle dogs. Int. J. Nanomed. 2015, 10, 3533-3545.

53. Su, Y.; Liu, M.; Xiong, Y.; Ding, J.; Liu, X.; Song, Y.; Deng, Y. Effects of stability of PEGylated micelles on the accelerated blood clearance phenomenon. Drug Deliv. Transl. Res. 2019, 9, 66-75. [CrossRef] [PubMed]

54. Liu, M.; Chu, Y.; Liu, H.; Su, Y.; Zhang, Q.; Jiao, J.; Liu, M.; Ding, J.; Liu, M.; Hu, Y.; et al. Accelerated blood clearance of nanoemulsions modified with PEG-cholesterol and PEG-phospholipid derivatives in rats: The effect of PEG-lipid linkages and PEG molecular Weights. Mol. Pharm. 2020, 17, 1059-1070. [CrossRef] [PubMed]

(C) 2020 by the authors. Licensee MDPI, Basel, Switzerland. This article is an open access article distributed under the terms and conditions of the Creative Commons Attribution (CC BY) license (http://creativecommons.org/licenses/by/4.0/). 